\title{
Alpha-fetoprotein (AFP) Elevation Gastric Adenocarcinoma and Importance of AFP Change in Tumor Response Evaluation
}

\author{
Ali Murat Tatlı ${ }^{*}$, Zuhat Urakcı ${ }^{2}$ Mehmet Emin Kalender ${ }^{3}$, Harun Arslan ${ }^{4}$, \\ Didem Taştekin ${ }^{5}$, Mehmet Ali Kaplan ${ }^{2}$
}

\begin{abstract}
Background: Elevated serum alpha-fetoprotein (AFP) levels in adults are considered abnormal. This parameter is used mostly in the diagnosis and follow-up of hepatocellular carcinomas and yolk sac tumors. Among the other rare tumors accompanied with elevated serum AFP levels, gastric cancer is the most common. In this study, we evaluated the follow-up and comparison of the treatment and marker response of patients with metastatic gastric cancer who had elevated serum AFP levels. Materials and Methods: We performed a retrospective study, including all consecutive patients with advanced gastric cancer, who received systemic chemotherapy with elevated AFP level. Results: Seventeen metastatic gastric cancer patients with elevated AFP levels at the time of diagnosis were evaluated. Fourteen $(82.4 \%)$ were males and three $(\mathbf{1 7 . 6 \%})$ were females. The primary tumor localization was the gastric body in $8(76.4 \%)$, cardia in $7(41.2 \%)$, and antrum in $2(11.8 \%)$. Hepatic metastasis was observed in $13(76.4 \%)$ at the time of diagnosis. When the relationship of AFP levels and carcinoembryonic antigen (CEA) response of the patients with their radiologic responses was evaluated, it was found that the radiologic response was compatible with AFP response in $16(94.1 \%)$ patients and with CEA response in $12(70.6 \%)$; however, in 5 (29.4\%) patients no accordance was observed between radiological and CEA responses. Conclusions: Followup of AFP levels in metastatic gastric cancer patients with elevated AFP levels may allow prediction of early treatment response and could be more useful than the CEA marker for follow-up in response evaluation.
\end{abstract}

Keywords: Alpha-fetoprotein - chemotherapy - gastric cancer - CEA

Asian Pac J Cancer Prev, 16 (5), 2003-2007

\section{Introduction}

Elevated serum alpha-fetoprotein (AFP) levels are often detected as a product of fetal liver, yolk sac, and some fetal gastrointestinal cells and decrease rapidly after birth (Gitlin et al., 1972). Among adults, however, elevated AFP levels are often considered abnormal. It is generally used as a marker in the diagnosis and follow-up of hepatocellular carcinomas and yolk sac tumors. While gastric cancer is the most common type of other cancers that might be accompanied by high serum AFP levels, in rare occasions, AFP elevation might also be seen in patients with tumors of other organs such as ovary, lungs, pancreas, esophagus, papilla vateri, colon, urinary bladder, uterus and the renal pelvis (Saito et al., 1989; Hocking et al., 1995; Ueno et al., 2001; Trompetas et al., 2003; Yamagata et al., 2004; Hamanakaet al., 2008; Isonishi et al., 2009). Bourreille et al., (1970) reported the first case of gastric cancer with liver metastasis and elevated AFP levels. In later studies that evaluated different case series of widespread and early stage gastric cancer, those were described as tumors with poor prognoses with high rates of liver metastasis and lymphatic and venous micro invasion (Chang et al., 1990; Motoyama et al., 1993; Shibata et al., 2007). Elevated AFP levels are reported likely to be observed in 1.3-15\% of all gastric cancer patients (McIntire et al., 1971;Akai and Kato, 1973; Takahashi et al., 1987). AFP-producing gastric cancer patients were determined to have significantly shorter survival than AFP-negative patients (Chang et al., 1992; Mittal et al., 2013). On the other hand, though AFP elevation often accompanies gastric cancer patients with hepatoid differentiation, it should not be expected to be high in all cases. Adenocarcinomas with hepatoid differentiation may be observed in the absence of elevated AFP levels as well. Nagai et al. reported that among the AFP-producing gastric cancer cases, those with hepatoid adenocarcinomas have worse prognoses than non-hepatoid cases (Nagai et al., 1993). Currently, there is no information of a difference between AFP-producing and AFP-negative gastric cancer patients in terms of treatment and follow-up in routine practice. In this study, the general characteristics along

Department Oncology Medical, ${ }^{1}$ Van Training and Research Hospital, Van, ${ }^{2}$ Dicle University, Medical Faculty, Diyarbakır, ${ }^{3}$ Gaziantep University, Medical Faculty, Gaziantep, ${ }^{5}$ IstanbulUniversity, Medical Faculty, Istanbul, ${ }^{4}$ Department Radiology, Van Training and Research Hospital, Van, Turkey*For correspondence: alimurattat@hotmail.com 
with their treatment response and marker follow-up of metastatic gastric cancer patients with elevated AFP levels, who were administered systemic chemotherapy, were evaluated.

\section{Materials and Methods}

In this study, we retrospectively reviewed the files of the patients diagnosed with metastatic gastric carcinoma, who received chemotherapy. The medical files and electronic records of the patients followed up between June 2011 and September 2014 in two centers were evaluated. All patients had gastric cancer confirmed histopathologically, and among them, only the AFP-producing group at metastatic stage who was at the beginning of their treatment was included in the study $(n=17)$. Patients with chronic hepatitis, cirrhosis, or hepatocellular carcinomas were excluded from study. Gender, age, tumor localization, metastatic areas, AFP and CEA values at the time of the diagnosis and post-treatment period, first-line therapy response, and survival rates were recorded. The marker responses were evaluated as a complete response if the baseline values returned to normal on the 4th post-chemotherapy week; partial response if more than $50 \%$ decrease was observed; stable response if the decrease was $<50 \%$ or an increase occurred; and progression if $>50 \%$ increase was observed in the marker level (Yamao et al., 1999; Riaz et al., 2009). Radiologic evaluations were made based on Response Evaluation Criteria in Solid Tumors (RECIST) version 1.1.

\section{Statistical analysis}

Statistical analyses were performed using Statistical Package for Social Sciences (SPSS) version 18. Descriptive statistical analysis was performed for the demographic characteristics of the patients.

\section{Results}

Investigation of the medical files and electronic records of metastatic gastric cancer patients yielded that AFP values of 89 patients were asked for at the time of diagnosis, and $17(19.1 \%)$ of them were determined to have elevated AFP levels. It was observed that AFP and CEA levels were monitored together in the follow-up of the patients. The median age of the patients was 59 (range:45-77) years. The numbers of male and female patients included in the study were $14(82.4 \%)$ and 3 $(17.6 \%)$, respectively. Primary tumor localizations were the gastric body in $8(47.1 \%)$, cardia in $7(41.2 \%)$, and antrum in $2(11.8 \%)$ of the cases. While metastasis regions included liver, peritoneum, lymph nodes, and lungs, 13 (76.4\%) of the patients had liver metastasis at the time of diagnosis. Well-differentiated tumors were not observed in any of the participants; while $8(47.1 \%)$ patients had poorly-differentiated and $9(52.9 \%)$ had moderatelydifferentiated adenocarcinoma. The general characteristics of our patients were demonstrated in Table 1 .

The highest and the lowest AFP levels observed among our patients at the time of diagnosis were $140080 \mathrm{ng} / \mathrm{ml}$ and $140 \mathrm{ng} / \mathrm{ml}$, respectively. Elevated AFP levels were accompanied by elevated CEA levels in all the cases except for one patient. Treatment response and AFP response were fully consistent in 16 of the 17 patients. The patient with the highest AFP level of $140080 \mathrm{ng} / \mathrm{ml}$ was determined to have their AFP level regress to 5600 $\mathrm{ng} / \mathrm{ml}$ within 2 weeks of treatment and to 1000 at the end of the $4^{\text {th }}$ week. Consistent with this marker response, an objective response was observed in the computerized tomography (CT) scan obtained at the end of the 4th week (Figure 1). There were 7 patients with regular monitoring of AFP and CEA before and after each treatment cycle. Figure 2 displays the treatment and AFP response rates of these patients. One of them who had marker progression by the beginning of $2^{\text {nd }}$ cycle was radiologically determined to be non-responsive, while the other 6 patients who had a marker response by the beginning of $2^{\text {nd }}$ cure were observed to be responsive radiologically, in accordance with their early marker responses. Treatment response rates of the patients were determined as 12 (70.6\%) partial responses, 2 (11.8\%) stable disease, and $3(17.6 \%)$ progression. Among 8 of the 12 patients that had progression following first-line treatment received second-line chemotherapy; 2 patients had a partial one

Table 1. Clinical and Pathologic Characteristics

\begin{tabular}{|c|c|c|c|c|c|}
\hline Case & Age & Gender & Tumour location & Distant metastasis location & Histological type \\
\hline 1 & 70 & M & Body & Liver-Lung & Poorly differentiated adenocarcinoma \\
\hline 2 & 54 & M & Body & Liver-Lymphnodes & Poorly differentiated adenocarcinoma \\
\hline 3 & 47 & M & Antrum & Liver-Lymphnodes & Moderately differentiated adenocarcinoma \\
\hline 4 & 51 & M & Cardia & Liver-Lymphnodes & Poorly differentiated adenocarcinoma \\
\hline 5 & 58 & M & Body & Lymphnodes & Poorly differentiated adenocarcinoma \\
\hline 6 & 75 & M & Cardia & Lymphnodes & Moderately differentiated adenocarcinoma \\
\hline 7 & 67 & $\mathrm{~F}$ & Cardia & Peritoneal-Lymphnodes & Moderately differentiated adenocarcinoma \\
\hline 8 & 63 & M & Body & Liver-Lymphnodes & Poorly differentiated adenocarcinoma \\
\hline 9 & 59 & M & Body & Liver & Poorly differentiated adenocarcinoma \\
\hline 10 & 54 & $\mathrm{M}$ & Body & Liver-Lymphnodes & Poorly differentiated adenocarcinoma \\
\hline 11 & 53 & M & Cardia & Liver-Lymphnodes & Poorly differentiated adenocarcinoma \\
\hline 12 & 73 & M & Body & Liver & Moderately differentiated adenocarcinoma \\
\hline 13 & 51 & M & Cardia & Liver & Moderately differentiated adenocarcinoma \\
\hline 14 & 68 & $\mathrm{~F}$ & Cardia & Liver & Moderately differentiated adenocarcinoma \\
\hline 15 & 77 & M & Antrum & Lymphnodes & Moderately differentiated adenocarcinoma \\
\hline 16 & 45 & $\mathrm{~F}$ & Body & Liver-Lymphnodes & Moderately differentiated adenocarcinoma \\
\hline 17 & 70 & M & Cardia & Liver-Lymphnodes & Moderately differentiated adenocarcinoma \\
\hline
\end{tabular}


Table 2. Serum Alpha-fetoprotein (AFP) Levels and Treatment Outcome Relationship

\begin{tabular}{|c|c|c|c|c|c|c|}
\hline Case & $\begin{array}{l}\text { Initial AFP } \\
\text { (ng/ml) }\end{array}$ & $\begin{array}{l}\text { AFP after treatment } \\
(\mathrm{ng} / \mathrm{ml})\end{array}$ & $\begin{array}{l}\text { Initial CEA } \\
\text { (ng/ml) }\end{array}$ & $\begin{array}{l}\text { CEA after reatment } \\
(\mathrm{ng} / \mathrm{ml})\end{array}$ & Response to treatment & $\begin{array}{l}\text { Outcome } \\
\text { (months) }\end{array}$ \\
\hline 1 & 590 & 6 & 13172 & 643 & Partial & 9, Died \\
\hline 2 & 218 & 90 & 91 & 68 & Partial & 10, Died \\
\hline 3 & 429 & 8 & 179 & 52 & Partial & 14, Alive \\
\hline 4 & 1000 & 22 & 728 & 9 & Partial & 9.5, Died \\
\hline 5 & 143 & 415 & 20 & 62 & Progression & 13, Died \\
\hline 6 & 565 & 96 & 5 & 4.5 & Partial & 10, Died \\
\hline 7 & 258 & 26 & 17 & 11 & Partial & 8.5, Died \\
\hline 8 & 16331 & 1100 & 150 & 6 & Partial & 8, Died \\
\hline 9 & 30000 & 50000 & 510 & 592 & Progression & 3, Died \\
\hline 10 & 6836 & 8 & 11.5 & 3.2 & Partial & 14.5, Died \\
\hline 11 & 17550 & 256 & 12.4 & 6.7 & Partial & 22, Died \\
\hline 12 & 200 & 150 & 9 & 8 & Stable & 11, Died \\
\hline 13 & 36900 & 16200 & 9 & 6 & Stable & 7, Died \\
\hline 14 & 187 & 47 & 52 & 8 & Partial & 15, Died \\
\hline 15 & 1210 & 5250 & 31 & 47 & Progression & 6, Died \\
\hline 16 & 140080 & 1000 & 38 & 9 & Partial & 7, Alive \\
\hline 17 & 140 & 10 & 25 & 11 & Partial & 7, Alive \\
\hline
\end{tabular}

Table 3. Comparisons of Responses with Tumor Markers and Radiology

\begin{tabular}{|c|c|c|c|c|}
\hline \multicolumn{2}{|c|}{$\begin{array}{l}\text { Case AFP } \\
\text { response }\end{array}$} & \multirow{2}{*}{$\begin{array}{c}\text { CEA } \\
\text { response } \\
\text { Partial }\end{array}$} & \multirow{2}{*}{$\begin{array}{c}\begin{array}{c}\text { Radiologic } \\
\text { response } \\
\text { (Recist 1.1) }\end{array} \\
\text { Partial }\end{array}$} & \multirow{2}{*}{$\begin{array}{c}\begin{array}{c}\text { Correlation with } \\
\text { radiologic } \\
\text { response }\end{array} \\
\text { AFP and CEA }\end{array}$} \\
\hline 1 & Partial & & & \\
\hline 2 & Partial & Stable & Partial & $\mathrm{AFP}$ \\
\hline 3 & Partial & Partial & Partial & $\mathrm{AFP}$ and CEA \\
\hline 4 & Partial & Partial & Partial & $\mathrm{AFP}$ and $\mathrm{CEA}$ \\
\hline 5 & Progression & Progression & Progression & $\mathrm{AFP}$ and CEA \\
\hline 6 & Partial & Stable & Partial & AFP \\
\hline 7 & Partial & Stable & Partial & AFP \\
\hline 8 & Partial & Partial & Partial & $\mathrm{AFP}$ and $\mathrm{CEA}$ \\
\hline 9 & Progression & Stable & Progression & AFP \\
\hline 10 & Partial & Partial & Partial & $\mathrm{AFP}$ and CEA \\
\hline 11 & Partial & Stable & Partial & $\mathrm{AFP}$ \\
\hline 12 & Stable & Stable & Stable & $\mathrm{AFP}$ and $\mathrm{CEA}$ \\
\hline 13 & Partial & Stable & Stable & CEA \\
\hline 14 & Partial & Partial & Partial & $\mathrm{AFP}$ and $\mathrm{CEA}$ \\
\hline 15 & Progression & Progression & Progression & $\mathrm{AFP}$ and CEA \\
\hline 16 & Partial & Partial & Partial & $\mathrm{AFP}$ and $\mathrm{CEA}$ \\
\hline 17 & Partial & Partial & Partial & AFP and CEA \\
\hline
\end{tabular}

had a stable disease; one was not evaluated for a response, and 4 patients had progression. AFP marker response and treatment response were compatible after second-line treatment as well. Treatment response, marker response, and survival rates of the patients are presented in Table 2. An evaluation of radiological and marker responses has shown that AFP marker response is consistent with the radiological response more strongly than CEA marker.

A consistency was detected between the radiological and AFP marker responses in 16 of the 17 patients evaluated in this study. Only one patient had a partial AFP marker response despite a stable disease upon radiological examination. On the other hand, CEA marker response evaluation has shown a consistency with the radiological response of 12, but not in five patients. A stable CEA marker response was observed in four patients with partial response and one patient with progression upon radiological evaluation. Consistency rates of marker and radiologic responses are displayed in Table 3.
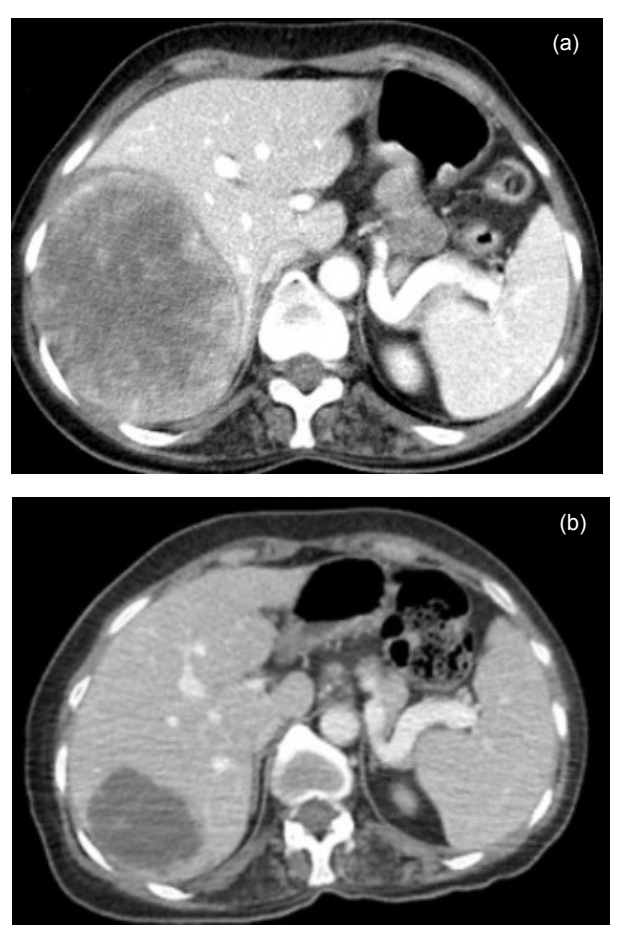

Figure 1. Axial CT. a) after IV contrast material in the right liver lobe marked contrast enhancing $123 \times 90 \mathrm{~mm}$ solid mass lesion (initial AFP level $140080 \mathrm{ng} / \mathrm{ml}$ ); b), $45 \times 37 \mathrm{~mm}$ cystic solid mass lesion (4 weeks after treatment, AFP $1000 \mathrm{ng} / \mathrm{ml}$ )

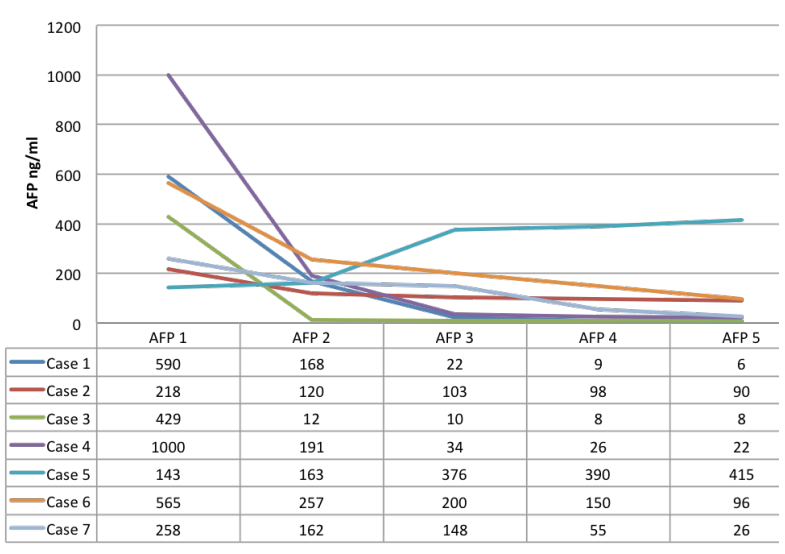

Figure 2. AFP Response after Treatment 


\section{Discussion}

The patients included in this study were metastatic gastric cancer patients with elevated serum AFP levels, which were followed-up at 2 centers in the Eastern Turkey. The relationship between elevated AFP levels was evaluated; and treatment response and the treatment responses by early decrease or increase in AFP levels were compared. AFP is a protein with a molecular weight of 70000 Dalton and a half-life of 5-7 days (Marx et al., 2002; Shu et al., 2012). Therefore, with its short half-life, it is believed to be an early indicator of treatment response.

In our study, as shown in Figure 1,AFP level follow-up was considered an early indicator of treatment response and disease progression in 7 patients. We observed a consistency between the radiological and AFP responses in all patients in this study, except for one patient. Largerscale studies often compared patients with high and normal AFP levels in a preoperative period. In those studies, as opposed to our study, the majority of the patients consisted of early stage patients with low rates of elevated AFP levels. Elevated AFP levels were detected in $58(4.5 \%)$ of the 1294 patients evaluated preoperatively in Lin et al.'s study, in $53(1.6 \%)$ of the 3374 patients in Inoue et al.'s study, and in 104 (2.3\%) of the 4426 patients in Liu et al.'s study (Inoue et al., 2010; Liu et al., 2010; Lin et al., 2014). Inoue et al. (2010)'s study with the relatively lower rates is believed to be affected by the high rate of early stage patients enrolled. We, on the other hand, evaluated metastatic gastric cancer patients only. Among the 89 patients whose AFP levels were evaluated, 17 $(19.1 \%)$ were determined to have elevated AFP levels, the highest proportion reported in medical literature so far. We believe that this difference may be caused by two reasons. First, all patients enrolled in our study were metastatic patients, which may have led to higher rates of elevated AFP levels. Second, the study was conducted in an area of Turkey that is different than the rest of the country as it is endemic for gastrointestinal system tumors, especially for gastric and esophagus cancers, with gastric cancer as the most common cancer type (Turkdogan et al., 1998; Baek et al., 2011).

Another distinct characteristic of our sample that is different than the literature is the tumor localization. Prior studies have reported antrum (the lower one-third of the stomach) as the most common (40\%-61.5\%) primary tumor localization (Inoue et al., 2010; Liu et al., 2010; Baek et al., 2011; Chun and Kwon, 2011; Lin et al., 2014). In our study, on the other hand, antrum was the least common tumor localization with $2(11.8 \%)$ cases. While different 5 -year survival rates $(9-66 \%)$ have been reported in many studies, the difference between these rates was believed to be primarily due to the difference between disease stages (Inoue et al., 2010; Liu et al., 2010; Lin et al., 2014; Chun and Kwon, 2011; Zhang et al., 2011). Maruyama et al. (2006), in their study comparing a large number of metastatic gastric cancer patients, reported that 5 -year survival was worst among early stage operated patients with elevated AFP levels, while stage 4 patients did not demonstrate a difference by high or normal AFP levels (8 vs 9.9\%).
The limitations of our study are that it was a retrospective study and was conducted with a regional homogeneous patient group. Lack of immunohisto chemical AFP staining in pathology specimens of our patients and of reporting on their hepatoid differentiation can also be listed among limitations. Elevated AFP levels have been reported not to cause a difference among stage 4 patients (Maruyama et al., 2006) but to have poor prognosis among hepatoid carcinoma cases compared to non-hepatoid cases (Nagai et al., 1993). In our study, independent of elevated AFP levels, partial response was observed among elevated AFP level patients as well, whose treatment responses are presented in Table 2. Among the 17 patients, total response rate for first-line therapy was $82.4 \%$ with $12(70.6 \%)$ partial responses and $2(11.8 \%)$ stable disease.

Metastatic gastric cancer patients with elevated AFP levels were also observed to have high CEA levels, and it was determined that both markers could be monitored for a treatment response. However, as shown in Table 3, radiological and marker response evaluation has shown that AFP marker response is more strongly consistent with the radiological response than is CEA marker response. Of the 17 patients, 16 (94.1\%) had AFP responses were consistent, but $12(70.6 \%)$ had CEA responses consistent with radiological responses. Therefore, AFP marker follow-up is considered of more value than CEA marker follow-up for metastatic gastric cancer patients with elevated AFP levels. Moreover, it should be considered that the short half-life of AFP could ensure early predictions of treatment response.

In conclusion, while regional differences are expected to have played a role, due to the high response rates obtained with our sample, it is concluded that the diseases might have an aggressive course for metastatic gastric cancer patients with elevated AFP levels and that AFP levels are a better indicator than CEA for early response and diagnosis to ensure early onset of treatment and patient follow-up.

\section{References}

Akai S, Kato K (1973). Serum $\alpha$-fetoprotein-positive stomach cancer. Gann Monogr, 14, 149-54.

Baek SK, Han SW, Oh DY, et al (2011). Clinic pathologic characteristics and treatment outcomes of hepatoid adenocarcinoma of the stomach, a rare but unique subtype of gastric cancer. BMC Gastroenterol, 11, 1-7.

Bourreille J, Metayer P, Sauger F, et al (1970). Existence of alpha feto protein during gastric-origin secondary cancer of the liver. Presse Med, 78, 1277-8.

Chang YC, Nagasue N, Abe S, et al (1990). The characteristics of AFP-producing early gastric cancer. J Jpn Surg Soc, 91, 1574-80.

Chang YC, Nagasue N, Abe S, et al (1992). Comparison between the clinicopathologic features of AFP-positive and AFPnegative gastric cancers. Am J Gastroenterol, 87, 321-5.

Chun H, Kwon SJ (2011). Clinic pathological characteristics of alpha-fetoprotein-producing gastric cancer. J Gastric Cancer, 11, 23-30.

Gitlin D, Pericelli A, Gitlin GM (1972). Synthesis of-fetoprotein by liver, yolk sac, and gastrointestinal tract of the human conceptus. Cancer Res, 32, 979-82. 
Hamanaka W, Yoneda S, Shirakusa T, et al (2008). Alphafetoprotein (AFP)-producing adrenocortical carcinoma-long survival with various therapeutic strategies including a lung resection: Report of a case. Surg Today, 38, 275-8.

Hocking GR, Shembrey M, Hay D, et al (1995). Alphafetoprotein- producing adenocarcinoma of the sigmoid colon with possible hepatoid differentiation. Pathology, 27, 277-9.

Inoue M, Sano T, Kuchiba A, et al (2008). Long-term results of gastrectomy for $\alpha$-fetoprotein-producing gastric cancer. $\mathrm{Br}$ J Surg, 97, 1056-61.

Isonishi S, Ogura A, Kiyokawa T, et al (2009). Alpha-fetoprotein (AFP)- producing ovarian tumor in an elderly woman. Int J Clin Oncol, 14, 70-3.

Lin HJ, Hsieh Y.H, Fang WL, et al (2014). Clinical manifestations in patients with alpha-fetoprotein-producing gastric cancer. Curr Oncol, 21, 394-9.

Liu X, Cheng Y, Sheng W, et al (2010). Analysis of clinicopathologic features and prognostic factors in hepatoid adenocarcinoma of the stomach. Am J SurgPathol, 34, 1465-71.

Liu X, Cheng Y, Sheng W, et al (2010). Clinicopathologic features and prognostic factors in alpha-fetoproteinproducing gastric cancers: analysis of 104 cases. J Surg Oncol, 102, 249-55.

Maruyama K, Kaminishi M, Hayashi K, et al (2006). Gastric cancer treated in 1991 in Japan: data analysis of nationwide registry. Gastric Cancer, 9, 51-66.

Marx GM, Boyce A, Goldstein D (2002). Elevated $\alpha$-fetoprotein and hepatic metastases-it's not always what it seems! Ann Oncol, 13, 167-9.

McIntire KR, Waldmann TA, Moertel CG, et al (1971). Serum alpha-fetoprotein in patients with neoplastic carcinomas. $N$ Engl J Med, 285, 1060-1.

Mittal A, Gupta SP, Jha DK, et al (2013). Impact of various tumor markers in prognosis of gastric cancer. a hospital based study from tertiary care hospital of Kathmandu valley. Asian Pac J Cancer Prev, 14, 1965-7.

Motoyama T, Aizawa K, Watanabe H, et al (1993). Alphafetoprotein-producing gastric carcinomas: A comparative study of three different subtypes. Acta Pathol Jpn, 43, 654-61.

Nagai E, Ueyama T, Yao T, et al (1993). Hepatoid adenocarcinoma of the stomach. Cancer, 72, 1827-35.

Riaz A, Ryu RK, Kulik LM, et al (2009). Alpha-fetoprotein response after locoregional therapy for hepatocellular carcinoma: oncologic marker of radiologic response, progression, and survival. J Clin Oncol, 27, 5734-42.

Saito S, Hatano T, Hayakawa M, et al (1989). Studies on alphafetoprotein produced by renal cell carcinoma. Cancer, $\mathbf{6 3}$, 544-9.

Shibata Y, Sato K, Kodama M, et al (2007). Alpha-fetoproteinproducing early gastric cancer of the remnant stomach: Report of a case. Surg Today, 37, 995-9.

Shu J, Li CG, Liu YC, et al (2012). Comparison of serum tumor associated material (TAM) with conventional biomarkers in cancer patients. Asian Pac J Cancer Prev, 13, 2399-403.

Takahashi Y, Mai O, Ogino T, et al (1987). Clinicopathological study of AFP-producing gastric cancer. Significance of AFP in gastric cancer. J Jpn Surg Soc, 88, 696-700.

Trompetas V, Varsamidakis N, Frangia K, et al (2003). Gastric hepatoid adenocarcinoma and familial investigation: does it always produce alpha-fetoprotein? Eur J Gastroenterol Hepatol, 15, 1241-4.

Turkdogan MK, Akman N, Tuncer I, et al (1998). The high prevalence of esophageal and gastric cancers in Eastern Turkey. Med Biol Environ, 26, 79-84.

Turkdogan MK, Testereci H, Akman N, et al (2003). Dietary nitrate and nitrite levels in an endemic upper gastrointestinal (esophageal and gastric) cancer region of Turkey. Turk $J$ Gastroenterol, 14, 50-3.

Ueno M, Nakashima J, Ohigashi T, et al (2001). Establishment of a testicular carcinoma cell line producing alpha-fetoprotein. BJU Int, 88, 611-21.

Yamagata T, Yamagata Y, Nakanishi M, et al (2004). A case of primary lung cancer producing alpha-fetoprotein. Can Respir J, 11, 504-6.

Yamao T, Kai S, Kazami A, et al (1999). Tumor markers CEA, CA19-9 and CA125in monitoring of response to systemic chemotherapy in patients with advanced gastric cancer. Jpn $J$ Clin Oncol, 29, 550-5.

Zhang JF, Shi SS, Shao YF, et al (2011). Clinic pathological and prognostic features of hepatoid adenocarcinoma of the stomach. Chin Med J, 124, 1470-6. 